\title{
Sulfur nanoparticles transform montmorillonite into an inorganic surfactant applicable in thermoplastics processing
}

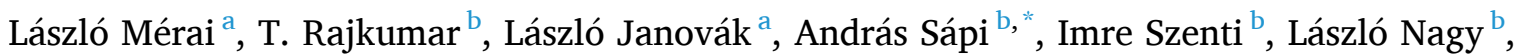 \\ Tamás Molnár $^{\mathrm{c}}$, István Bíró ${ }^{\mathrm{c}}$, József Sárosi ${ }^{\mathrm{c}}$, Ákos Kukovecz ${ }^{\mathrm{b}}$, Zoltán Kónya ${ }^{\mathrm{b}}$ \\ ${ }^{a}$ University of Szeged, Interdisciplinary Excellence Centre, Department of Physical Chemistry and Materials Science, H-6720, Rerrich Béla Tér 1, Szeged, Hungary \\ ${ }^{\mathrm{b}}$ University of Szeged, Interdisciplinary Excellence Centre, Department of Applied and Environmental Chemistry, H-6720, Rerrich Béla Tér 1, Szeged, Hungary \\ ${ }^{\mathrm{c}}$ University of Szeged, Faculty of Engineering, Department of Technology, H-6724, Mars Tér 7, Szeged, Hungary
}

\section{A R T I C L E I N F O}

\section{Keywords:}

Montmorillonite

Sulfur

Inorganic surfactant

High-density polyethylene

Melt blending

Tensile strength

\begin{abstract}
A B S T R A C T
We report on the modification of montmorillonite (MMT) with sulfur nanoparticles (S NPs) that yields a MMT/S nanocomposite exhibiting surfactant-like amphiphilic behavior at 80:20 MMT:S weight-to-weight ratio. The new material was successfully used as an inorganic surfactant for the dispergation of carbon black (CB) in HighDensity Polyethylene (HDPE). Both the novel inorganic surfactants and the HDPE-based nanocomposite products were characterized rigorously by XRD, TGA-DSC, SEM, TEM, zeta potential measurement, static contact angle measurement, tensile strength analysis, particle charge measurement, and X-ray micro-computed tomography. It was found that the presence of $0.5 \mathrm{wt} \% \mathrm{MMT} / \mathrm{S}$ has no adverse effects on HDPE product quality. On the contrary, the MMT/S additive improves the homogeneity of the distribution of CB particles in the HDPE matrix, which is very important because of the UV stabilization properties of CB. The industrial relevance of this finding is that it demonstrates how the right composite of two cheap and readily available inorganic materials can improve the properties of one of the most widely used thermoplastics.
\end{abstract}

\section{Introduction}

Montmorillonite (MMT) based polymer nanocomposites usually have enhanced mechanical, thermal, barrier and flame-retardant properties in comparison to pristine polymers [1,2]. MMT was extensively used as filler for preparing polymer/MMT nanocomposites due to their unique properties like nanometer scale thickness and high aspect ratio [3,4]. MMT is a 2:1 phyllosilicate mineral with two tetrahedral silica sheets that sandwich a central alumina octahedral sheet [5]. The effective dispersion of MMT filler into the polymer matrix is a key factor in determining the final properties of the polymer/MMT nanocomposites. High-density polyethylene (HDPE) is the most commonly used thermoplastics due to its regular chain structure, high melting temperature, excellent thermal and electrical resistance, good tensile strength, low cost and high abrasion resistance [6-8]. Unfortunately, HDPE is a hydrophobic viscously melted material, which leads to incompatibility while blending with MMT with hydrophilic properties. Therefore, it is very important to promote the adhesion between the HDPE and MMT, which can be done by either modifying HDPE or MMT.
However, modifying HDPE can result in obvious increase in cost and complexity of preparation process. Thus, modification of MMT is useful approach to improve the compatibility between MMT and HDPE. As MMTs are hydrophilic in nature, it is compatible with hydrophilic polymers. In order to be compatible with HDPE, the hydrophilic MMT lamellae must be hydrophobized. Commonly, this surface modification is done by ion exchange reactions with cationic surfactants such as alkyl-ammonium cations. These cations not only lower the surface energy of the clay but also provide functional groups that can react with the polymer. However, the marginal thermal stability of the alkyl-ammonium cations leads to the degradation of polymer/MMT nanocomposites $[9,10]$.

In this work, we prepared organophilic MMT by adding Sulfur as an inert and cheap inorganic surface modifier to the MMT lamellae. The surface adsorption of the Sulfur particles on the clay lamellae was carried out with negligible amount $(<0.03 \mathrm{wt} \%)$ of chitosan macromolecules and the obtained filler material with composition dependent hydrophobicity was blended in HDPE polymer matrix. The as-prepared MMT/HDPE composites were characterized by XRD, TEM, SEM, zeta

\footnotetext{
* Corresponding author.

E-mail address: sapia@chem.u-szeged.hu (A. Sápi).
} 
potential, static contact angles, TGA-DSC, tensile strength, particle charge detector and X-ray micro-computed tomography analysis. We found that, the CB additive of the HDPE was sufficiently dispersed by adding small amount of the inorganic MMT/S surfactants to the melted CB-containing HDPE during the blending process.

\section{Materials and methods}

\subsection{Preparation of sulfur hydropobized montmorillonite composite particles}

To obtain sulfur nanoparticles (S NPs) by a modified Raffo-method, $0.03 \mathrm{~g}$ of Sulfur powder (Sigma Aldrich) was dissolved in $4 \mathrm{ml}$ of 10 $\mathrm{M}$ aqueous $\mathrm{NaOH}$-solution (solution $\mathrm{pH}=14.15$ ) at a temperature of 80 ${ }^{\circ} \mathrm{C}$. Afterwards, the liquid was stirred and diluted with $1 \mathrm{ml}$ of $10 \mathrm{M}$ $\mathrm{NaOH}$ solution, then $4.65 \mathrm{ml}$ of $2.5 \mathrm{M} \mathrm{H}_{2} \mathrm{SO}_{4}$ solution was added dropwise until the initial solution became turbid ( $\mathrm{pH}=13.95$ at precipitation) due to the formation of sulfur nanoparticles $(\mathrm{c}=\sim 0.09 \mathrm{~g} /$ $100 \mathrm{ml})$.

The resulting precipitated sulfur was centrifuged ( $8000 \mathrm{rpm}, 5 \mathrm{~min}$ ), thoroughly washed with deionized water and dried overnight at $60{ }^{\circ} \mathrm{C}$. The S NPs were then redispersed in deionized water $(\mathrm{c}=0.1 \mathrm{~g} / 100 \mathrm{ml})$, then $200 \mu \mathrm{L}$ of $0.01 \mathrm{~g} / 100 \mathrm{ml}$ high molecular weight chitosan (Chit; $\mathrm{M}_{\mathrm{W}}$ $=310-375 \mathrm{kDa}$; Sigma) solution (dissolved in $0.13 \%$ aqueous aceticacid; $\mathrm{pH} \sim 4.3$ ) was added to the dispersion to turn the streaming potential to positive. To form MMT/S composites, different amounts of aqueous EXM-838 montmorillonite (Süd-Chemie AG) dispersion ( $\mathrm{c}=$ $0.1 \mathrm{~g} / 100 \mathrm{ml}$ ) with cation exchange capacity of $\sim 100 \mathrm{mmol} / 100 \mathrm{~g}$ was added to the dispersion of S NPs overcharged with Chit macromolecules. The S/MMT ratio was systematically changed $(0,20,40,50,60,80,85$, 90 and $100 \mathrm{wt} \% \mathrm{~S}$-content, respectively) in the preparation process.

For contact angle measurements $5 \times 5 \mathrm{~cm}^{2}$ inorganic layers with $1 \pm$ $0.05 \mathrm{mg} / \mathrm{cm}^{2}$ specific mass was prepared, consisting of the MMT/S composite nanoparticles. The aqueous dispersions were evenly sprayed on clean plain glass plates, using an R180-type airbrush spray gun (3 bar operating pressure) and $\mathrm{N}_{2}$ as nebulizing gas.

\subsection{Preparation of MMT/S loaded HDPE composites}

The MMT/S (20:80 wt\%) composite was incorporated into commercial polyethylene dyed by black carbon by extrusion in a Brabender single screw extruder with a loading of $0.5 \mathrm{w} \%$. The material was stirred for $1 \mathrm{~h}$ at $140^{\circ} \mathrm{C}$ and then dried for $24 \mathrm{~h}$. For CT, Tensil strength and TGA measurements, a reference sample was also prepared where $0.5 \mathrm{wt} \%$ of a physical mixture (mortared) of MMT and S with the same ratio (20:80\%) was added to the carbon dyed HDPE and stirred for $1 \mathrm{~h}$ at $140{ }^{\circ} \mathrm{C}$ and then dried for $24 \mathrm{~h}$. Throughout the text, the notations of the samples are $\boldsymbol{C}$-PE: carbon dyed HDPE; $\boldsymbol{C}$-PE-C composite-loaded carbon dyed HDPE; C-PE-P: physical mixture-loaded carbon dyed HDPE.

\subsection{Methods of sample characterization}

The size distribution and morphology of the prepared composite particles were studied with HRTEM: the applied FEI Tecnai G2 $20 \mathrm{X}$ TWIN microscope is equipped with a tungsten cathode $(200 \mathrm{kV}$ acceleration voltage).

Surface morphology of the MMT/S layers was examined with a SEM-Hitachi S-4700 field emission scanning electron microscope (secondary electron detector, acceleration voltage: 10 or $20 \mathrm{kV}$ ), while the surficial composition was studied using a Röntec EDS detector.

Surface charge values of the aqueous dispersion of the components were measured by means of a particle charge detector (PCD-04 Particle Charge Detector; Mütek Analytic GmbH, Germany) with manual titration. Under a titration process the negative surface charge of the MMT (c $=0.1 \%)$ and S NPs $(\mathrm{c}=0.1 \%)$ suspensions were compensated with positively charged Chit molecules $(\mathrm{c}=0.01 \%$; $\mathrm{pH} \sim 4.3$ ) in aqueous medium, with concomitant streaming potential measurements.

The zeta potential values of the $0.01 \mathrm{wt} \% \mathrm{MMT} / \mathrm{S}$ aqueous dispersions were determined by dynamic light scattering (DLS) with a Zetasizer Nano ZS ZEN 4003 apparatus (Malvern Ins., UK) equipped with a $\mathrm{He}-\mathrm{Ne}$ laser $(\lambda=633 \mathrm{~nm})$. The measurements were performed at $25 \pm$ $0.1{ }^{\circ} \mathrm{C}$. The measurements were carried out in triplicate, and average values are reported. Error bars refer to the standard deviation.

Composition-dependent crystalline properties were studied using a Philips powder X-ray-diffractometer (PW 1830 generator, PW 1820 goniometer, $\mathrm{CuK}_{\alpha}: \lambda=0.1542 \mathrm{~nm}, 40-50 \mathrm{kV}, 30-40 \mathrm{~mA}, 2 \theta: 2-70^{\circ}, 25.0$ $\left.\pm 0.5^{\circ} \mathrm{C}\right)$.

The apparent static contact angles on MMT/S layers were measured according to the sessile drop technique at $25.0 \pm 0.5{ }^{\circ} \mathrm{C}$ under atmospheric pressure, applying an EasyDrop drop shape analysator (Krüss $\mathrm{GmbH}$, Hamburg, Germany) equipped with DSA100 software, a Peltier temperature chamber and a steel syringe needle of $0.5 \mathrm{~mm}$ diameter, and using deionized water as test liquid.

The DSC curves of the MMT/S composites were recorded applying Mettler Toledo DSC822 $2^{\mathrm{e}}$ device. In the tests, the solid powder samples were heated from $25{ }^{\circ} \mathrm{C}$ to $150{ }^{\circ} \mathrm{C}$ and cooled down to $25^{\circ} \mathrm{C}$ again at a rate of $5^{\circ} \mathrm{C} \cdot \mathrm{min}^{-1}$ in sealed aluminium sample holders with a hole at the top. Dry compressed $\mathrm{N}_{2}$ was used as a carrier gas at a flow rate of 50 $\mathrm{mL} \cdot \mathrm{min}^{-1}$. The obtained thermoanalytical data was processed using STAR $^{\mathrm{e}}$ software.

The thermogravimetric analyses were performed on a TA Instruments TGA Q500 thermogravimetric analyzer equipped with a standard furnace. $1-5 \mathrm{mg}$ of the samples were analyzed in platinum pans at a heating rate of $10^{\circ} \mathrm{C} \cdot \mathrm{min}^{-1}$ to $600{ }^{\circ} \mathrm{C}$ in an atmosphere of air flowing at $60 \mathrm{~mL} \cdot \mathrm{min}^{-1}$.

The tensile tests were performed using a Galbadini Quasar 100 tensiometer according to the ISO 527 procedure, using dumbbells specimens with a gauge length of $108 \mathrm{~mm}$, a width of $10 \mathrm{~mm}$ and a thickness of $4 \mathrm{~mm}$. The stretching tests were performed at room temperature at $10 \mathrm{~mm} \cdot \mathrm{min}^{-1}$. All tests were carried out on a minimum of 5 samples and the reported results are average values.

The size distribution of carbon black particles in the HDPE matrix was obtained via X-ray micro-computed tomography analysis, which was conducted using the X-ray Microtomography equipment (Skyscan 2211). The sample dimension of $\sim 8.0 \times 2.0 \times 1.0 \mathrm{~mm}^{3}$ were scanned using $11 \mathrm{Mp}$ cooled CCD camera by applying the source voltage of $60 \mathrm{kV}$ and the source current $800 \mu \mathrm{A}$ with an exposure time of $650 \mathrm{~ms}$. The voxel size of these dataset was $1 \mu \mathrm{m}$. NRecon reconstruction software was used to reconstruct the projected images with the pixel size of 4032 $\times 2688$ and CTan and CTvol software were used to represent the 3D models. Important to note that the smallest particles what we see with CT under these parameters is $216 \mu \mathrm{m}^{3}$. Although aggregation of clay minerals is inevitable, we believe that they do not exceed $100 \mu \mathrm{m}^{3}$ thus these particles are invisible and CB particles are visible during CT measurement. Only particles larger than $6 * 6 * 6$ voxel were considered in the calculation.

For the optical characterization of polymer samples, diffuse reflectance UV-VIS spectra were recorded applying a CHEM2000 UV-VIS (USB2000+UV-VIS, Ocean Optics Inc) spectrophotometer, equipped with an integrating sphere.

\section{Results and discussion}

\subsection{Structural characterization of composite nanoparticles}

The hydrophobization of the different polymer filler materials with initial hydrophilic properties is a possible route to provide improved mechanical properties to polymer based composites through increasing compatibility between the hydrophobic polymer matrix and the initially hydrophilic filler materials [11]. In this study the EXM-838 MMT clay sheets as well-known polymer filler material was hydrophobized with cheap and easy-available inorganic elemental S. To achieve this goal, S 

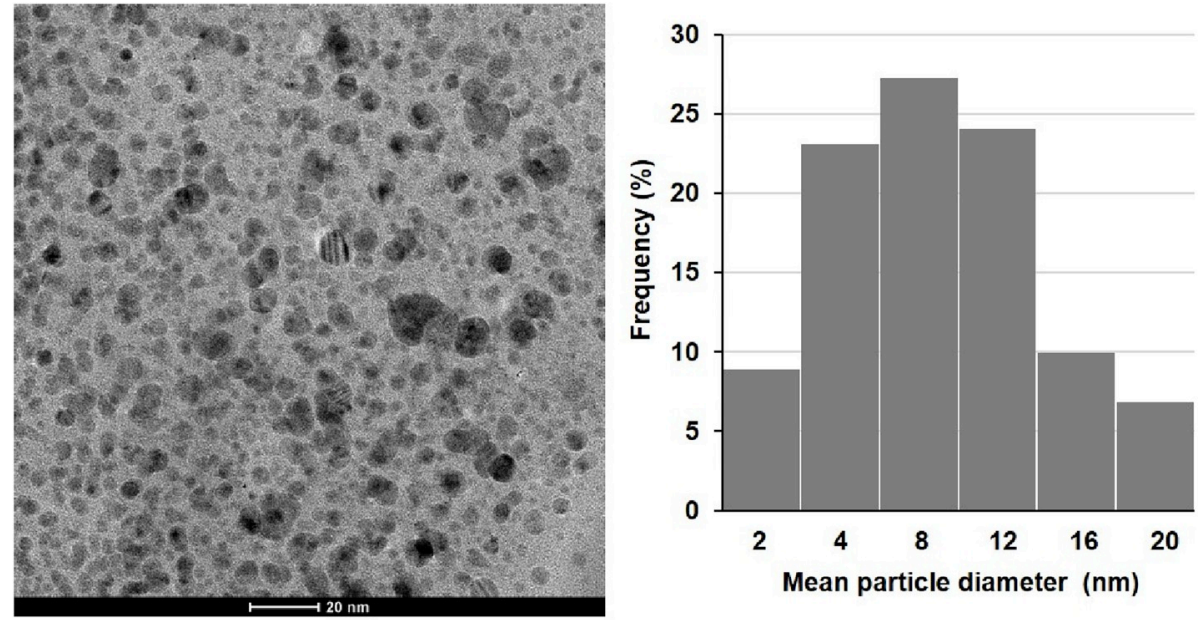

Fig. 1. TEM-micrographs of precipitated sulfur nanoparticles and the corresponding size-distribution of the synthetized sample.
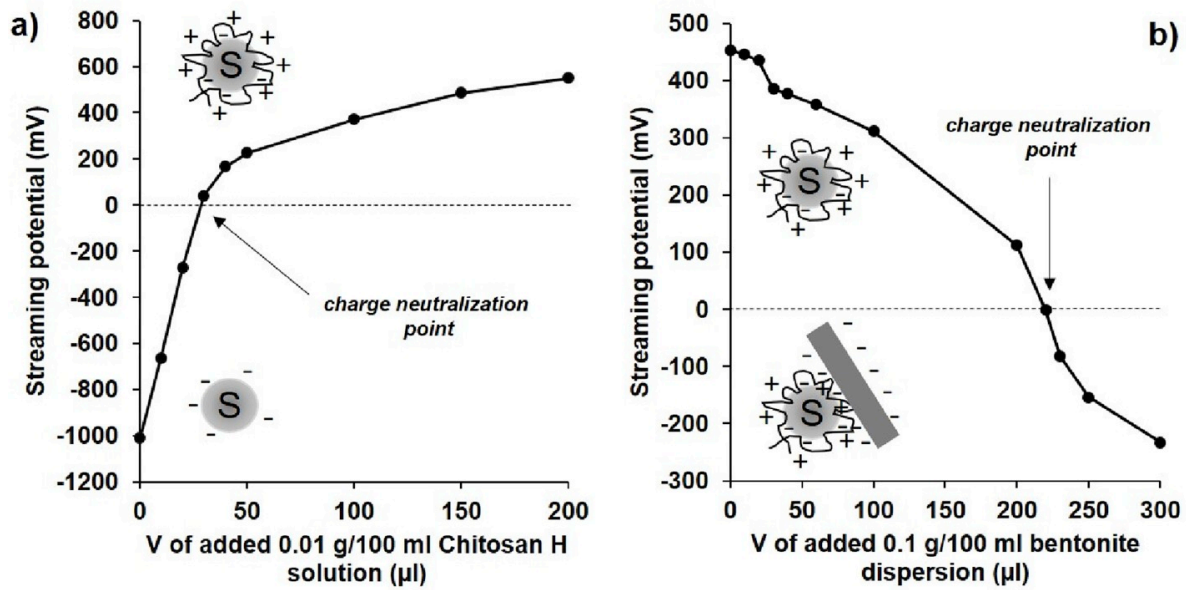

Fig. 2. The measured streaming potential values of the aqueous re-suspended $0.1 \%$ sulfur nanodispersion during the addition of $0.01 \%$ high molecular weight ( $\mathrm{M}_{\mathrm{w}}$ $=310000-375000 \mathrm{Da}$ ) chitosan solution (a.) and the streaming potential values of 0.1\% EXM-838 MMT dispersion during the addition of chitosan-overcharged sulfur nanoparticles ( $0.1 \%$ dispersion) (b).

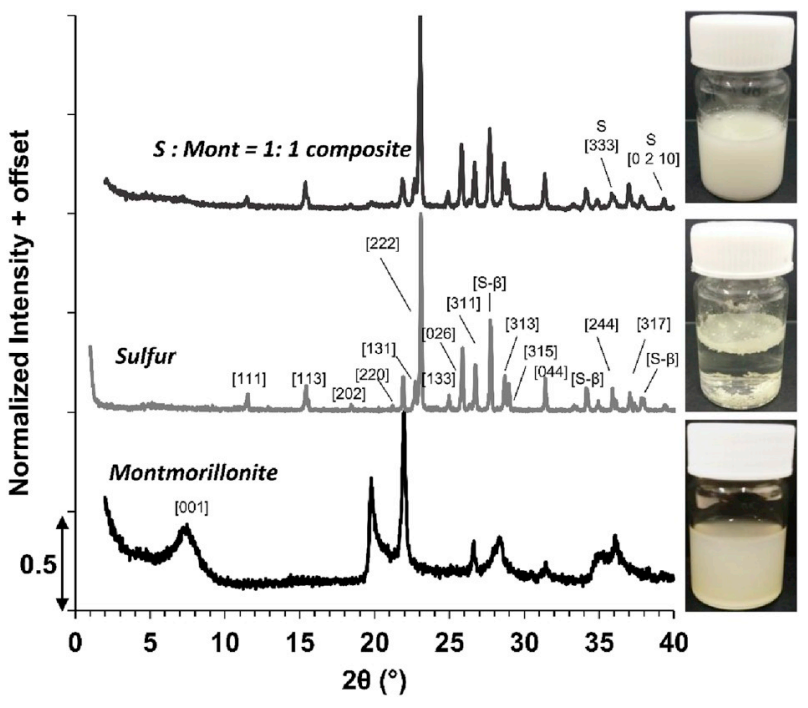

Fig. 3. Powder X-ray diffractograms and aqueous dispersions of initial EXM838 MMT, $S$ and their 1:1 composite.

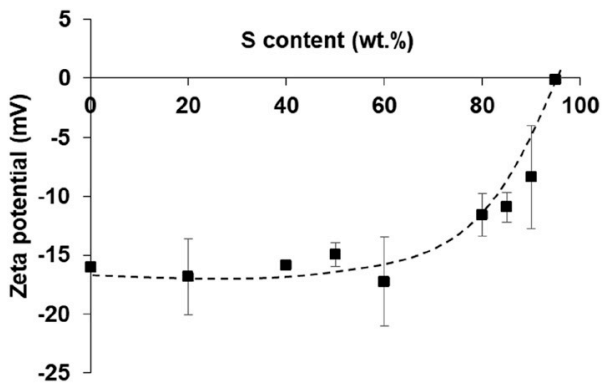

Fig. 4. Zeta potential values of $0.01 \mathrm{wt} \% \mathrm{MMT} / \mathrm{S}$ aqueous suspension with different S-content (the dashed line is a guide for the eyes only, it does not represent the actual trends).

NPs with a maximum diameter of $40 \mathrm{~nm}$ were synthesized applying a simple precipitation method. Despites there are many reported bottom-up routes to prepare S NPs, including e.g. the Weimarn [12], and Raffo-methods [13] or the sulfur vapour deposition on cold water [14], only the Raffo-methods offers particle yields high enough $(\sim 600 \mathrm{~g} / \mathrm{l})$ to be industrially profitable besides requiring more environmentally friendly aqueous media [15]. Our version of the applied Raffo-method is 


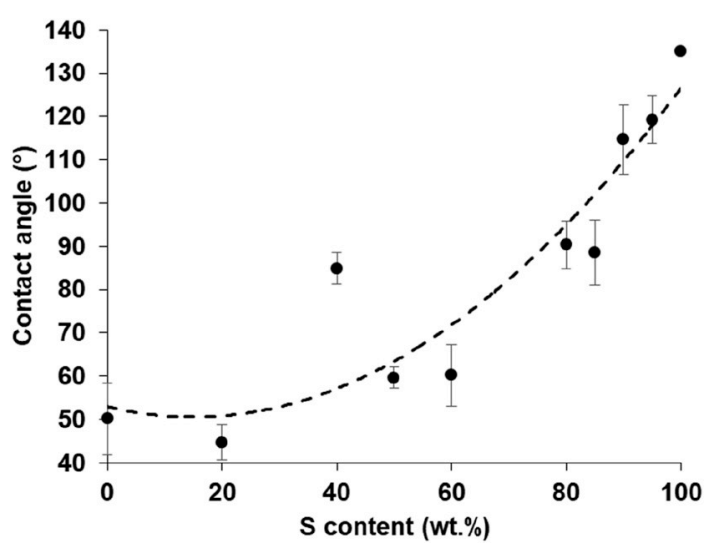

Fig. 5. The effect of $S$ content on the static water contact angle values of the $\mathrm{MMT} / \mathrm{S}$ composite powder layers.

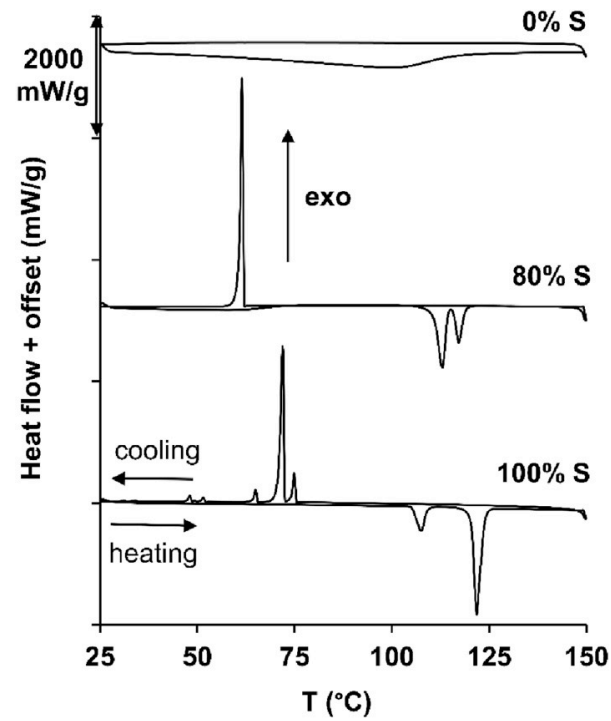

Fig. 6. DSC curves of initial MMT, S and the MMT/S with $80 \%$ of S.

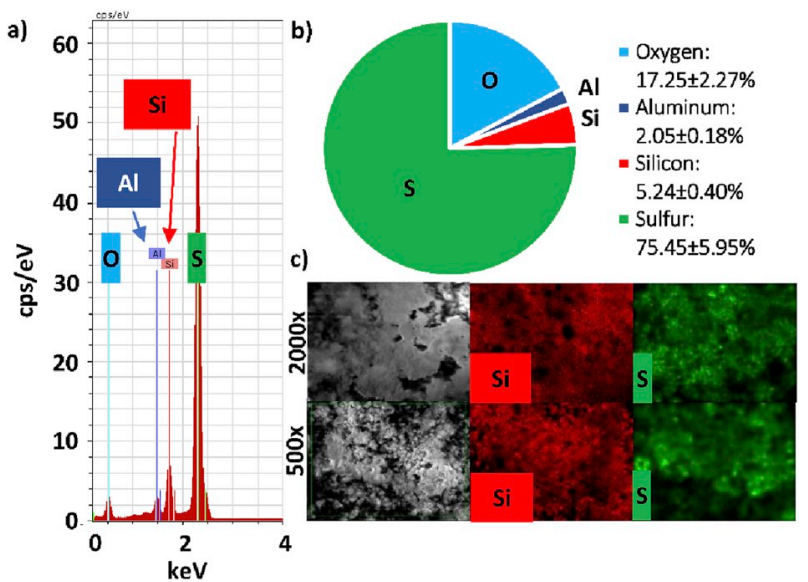

Fig. 7. EDX-spectra a) elemental composition chart (wt.\%) b) SEM micrographs with the corresponding magnifications and EDX-mapping c) of MMT/S composite with $80 \mathrm{wt} \%$ S-content.

based on the following chemical reactions15:

$4 \mathrm{~S}+6 \mathrm{NaOH}=\mathrm{Na} 2 \mathrm{~S} 2 \mathrm{O} 3+2 \mathrm{Na} 2 \mathrm{~S}+3 \mathrm{H} 2 \mathrm{O}$

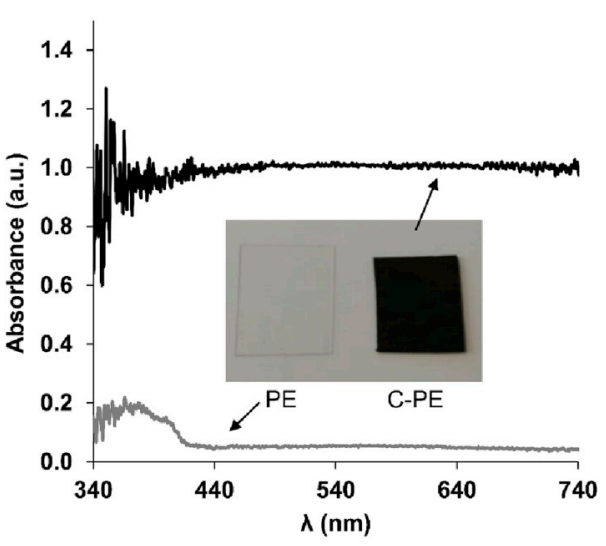

Fig. 8. UV-VIS diffuse reflectance spectra and the corresponding photographs of PE and C-PE samples.

$$
\begin{aligned}
& \mathrm{Na}_{2} \mathrm{~S}_{2} \mathrm{O}_{3}+\mathrm{H}_{2} \mathrm{SO}_{4}=\mathrm{H}_{2} \mathrm{~S}_{2} \mathrm{O}_{3}+\mathrm{Na}_{2} \mathrm{SO}_{4} \\
& \mathrm{x} \mathrm{H}_{2} \mathrm{~S}_{2} \mathrm{O}_{3}=\mathrm{y} \mathrm{H}_{2} \mathrm{O}+\mathrm{z} \mathrm{SO}_{2}+\mathrm{H}_{2} \mathrm{~S}_{\mathrm{m}} \mathrm{O}_{6} \\
& \mathrm{H}_{2} \mathrm{~S}_{\mathrm{m}} \mathrm{O}_{6}=\mathrm{H}_{2} \mathrm{~S}_{\mathrm{m}-\mathrm{n}} \mathrm{O}_{6}+\mathrm{S}_{\mathrm{n}}(\mathrm{n}>5)
\end{aligned}
$$

The core step of all Raffo-methods is the colloidal $\mathrm{S}$ formation, happening upon the acidification of $\mathrm{Na}_{2} \mathrm{~S}_{2} \mathrm{O}_{3}$ solutions with sulfuric acid (4. reaction step). We obtained our $\mathrm{S}_{2} \mathrm{O}_{3}^{2-}$-containing solution as a result of dissolving elemental $\mathrm{S}$ powder in $10 \mathrm{M} \mathrm{NaOH}$-solution at $80^{\circ} \mathrm{C}$, then $\mathrm{S}$-nanoparticles with a maximum mean diameter of $40 \mathrm{~nm}$ formed upon the following addition of $2.5 \mathrm{M} \mathrm{H}_{2} \mathrm{SO}_{4}$ solution. According to Equations (1)-(4), the dissolution of sulfur in such media is a disproportionation reaction, while the reverse precipitation reaction is a synproportionation. According to the literature [15], the x,y,z stochiometric coefficients can vary, depending on the reaction conditions, and the m-value is not well defined by them because polythionates with different chain-lengths can form in the reaction. However, in our case as we aim for the synthesis of sulfur nanoparticles the exact constitution of polythionates and sulfur homomolecules is less relevant and therefore were not studied in details.

As the TEM images and the corresponding histogram in Fig. 1 shows, the size distribution of the obtained spherical sulfur nanoparticles is relatively polydisperse. The mean diameters range between 1 and 40 $\mathrm{nm}$. This size is comparable to the size of the lamellar EXM-838 MMT lamellae with $\sim 2 \mathrm{~nm}$ thickness and lateral size of about few hundred nanometers.

The fixation of the S NPs on the surface of the MMT particles was executed exploiting electrostatic interactions. Despite elemental $\mathrm{S}$ does not contain ionic functionalities, Coehn's rule implies that the surface of low permittivity $S$ particles is slightly negatively charged in aquaeuos medium (point of zero charge at $\mathrm{pH}=2$ ). As MMT lamellae also have negative surface charge excess even in weak acidic media (point of zero charge at $\mathrm{pH}=4-5.9)[16,17]$ we used positively (in $\mathrm{pH}=4.3$ solution) charged chitosan biopolymer macromolecules (Chit; $\mathrm{Mw}=310-375$ $\mathrm{kDa}$ ) as cationic binding agent between the negatively charged inorganic components.

As the characteristic particle charge titration curve of Fig. 2a shows, the initial streaming potential $(-1010 \mathrm{mV})$ of the $0.1 \mathrm{wt} \% \mathrm{~S} \mathrm{NP}$ dispersion was continuously increased upon the addition of $0.01 \mathrm{wt} \%$ chitosan solution and at about $30 \mu \mathrm{l}$ added volume it was reached the 0 $\mathrm{mV}$ potential value (charge neutralization point). However, it also can be seen that the initially negatively charged S NPs can be overcharged by the further addition of the cationic polyelectrolyte. In the second step $0.1 \mathrm{wt} \%$ MMT aqueous dispersion was added dropwise to this overcharged $\mathrm{S}$ based dispersion resulting decreasing streaming potentials and the charge neutralization was occurred after the addition of $220 \mu \mathrm{l}$ 
A

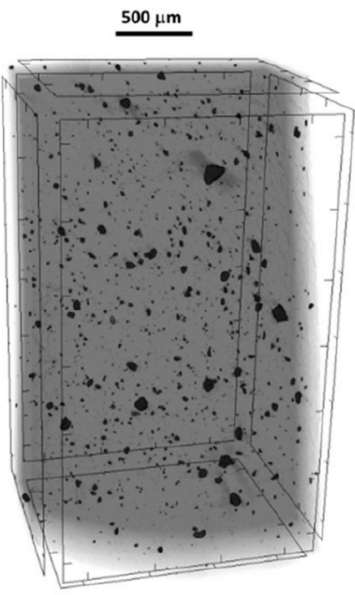

B

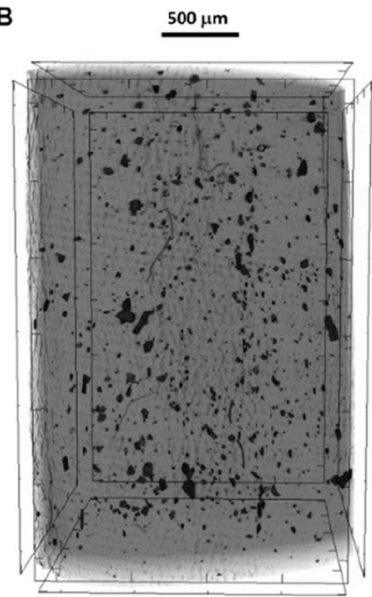

C

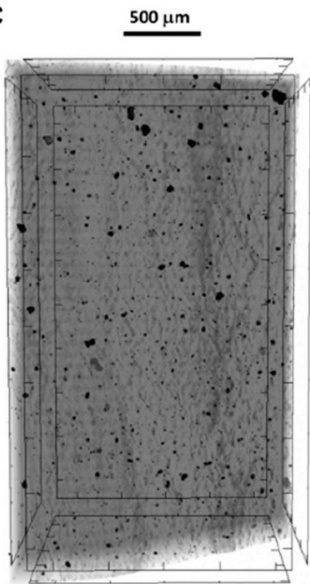

Fig. 9. 3D micro-CT image of C-PE (A), C-PE-P (B) and C-PE-C (C) samples.

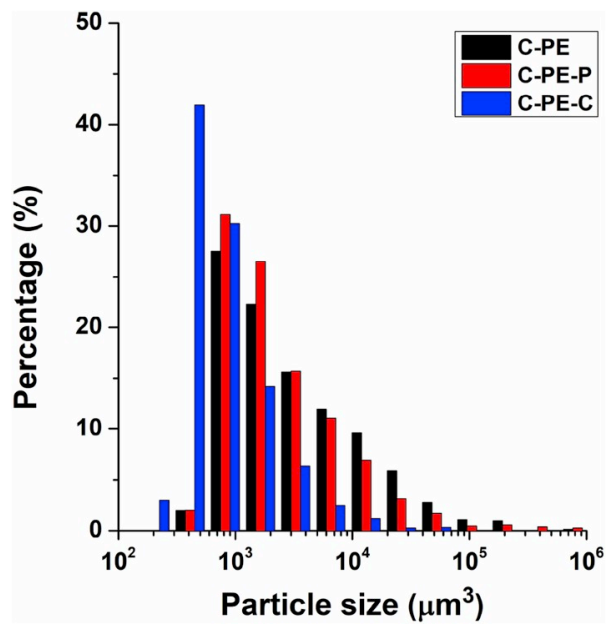

Fig. 10. Particle size distribution of carbon black particles in the polymer samples.

Table 1

CT image-based particle density values of the examined specimens.

\begin{tabular}{lll}
\hline Sample & Particle density (number of particle $/ \mathrm{mm}^{3}$ ) & Average particle size $\left(\mu \mathrm{m}^{3}\right)$ \\
\hline C-PE & 215 & 820 \\
C-PE-P & 154 & 820 \\
C-PE-C & 658 & 410 \\
\hline
\end{tabular}

MMT solution (Fig. 2b). It is important to note that considering the concentrations and added volumes of the components, the composite contains less than $0.03 \mathrm{wt} \%$ Chit polymer, which means that we could completely ignore the presence of Chit during sample characterization.

\subsection{Structural and interfacial properties}

In the previous chapter it was presented that the S NPs overcharged with negligible amount $(<0.03 \mathrm{wt} \%)$ of chitosan macromolecules could be successfully immobilized on the surface of MMT lamellae via electrostatic interactions. In the continuation, we systematically changed the ratio of the components to get samples with varied behaviors.

To study the crystalline structure of the resulted MMT/S composite powders, X-ray diffractograms were recorded. Fig. 3 shows the diffractograms of initial S, MMT and their 1:1 composite. As the sulfurcontent increases, the characteristic peaks of MMT at $2 \theta=7.54^{\circ}$
[001], $19.78^{\circ}, 22.20^{\circ}, 26.60^{\circ}, 28.32^{\circ}, 35.00^{\circ}$ and $36.08^{\circ}[18,19]$ decrease in intensity and disappear, while other peaks appear, attributed to the orthorhombic $\alpha$-sulfur (S) (with higher intensities: $23.10^{\circ}$ [222], $25.88^{\circ}$ [026], $26.76^{\circ}$ [311], 28.96 ${ }^{\circ}$ [313]) [20]. Moreover, some peaks are characteristics to monoclinic $\beta$-sulfur (S- $\beta$ ) $\left(27.74^{\circ}, 31.08^{\circ}, 37.84^{\circ}\right)$ [21]. The corresponding Miller-indices of the identifiable reflections are indicated in Fig. 3 however, in the case of MMT, only the [001] crystal plane was identified according to JCPDS card 29-1499 in the absence of other related literature. These observations indicate the crystalline nature of the S NPs, since no broad bands of amorphous phase were detected. As it can be seen in the inserted pictures of Fig. 3. S NPs are barely dispersible in water, but the addition of hydrophilic MMT resulted in better water dispersibility.

As the original reflections of MMT are barely identifiable in the XRD patterns of the composites, we propose complete or at least partial delamination for MMT. The interlayer spacing of unfunctionalized swollen MMT in aquaeuos medium ranges from 1 to $2 \mathrm{~nm}$ [22,23], and as Fig. 1 shows, a little less than $10 \%$ of the detectable particles have 2 $\mathrm{nm}$ or smaller mean diameters, therefore the intercalation of smaller polyelectrolyte-covered S NPs is theoretically possible.

Zeta potential measurements (Fig. 4.) showed that the initially lower negative zeta potential values $(\sim-16 \mathrm{mV})$ of MMT lamellae exceed 0 $\mathrm{mV}$ as the $\mathrm{S}$ content of the composites increases. The decrease in zeta potential also indicates the increasing S-coverage of clay lamellae.

The above presented results indicated that the presence of the sulfur nanoparticles changed the wetting and structural properties of MMT composite filler material which was also evidenced by contact angle measurements (Fig. 5). As the sulfur content increases, the spray-coated composite films (with $1 \pm 0,5 \mathrm{mg} / \mathrm{cm}^{2}$ specific layer mass) changed their wetting character from hydrophilic with water contact angle (WCA of $50.1^{\circ}$ at $0 \% \mathrm{~S} \mathrm{NPs}$ ) to hydrophobic (WCA $=130.0^{\circ}$ at $100 \% \mathrm{~S} \mathrm{NPs}$ ), with a sharp contact angle-increase at $80 \%$ S NP content.

This composition dependent wetting characteristics was also evidenced by DSC measurements. The DSC-curve of initial MMT in Fig. 6 shows a broad endothermic peak $\left(\mathrm{T}_{\text {end. }}=101.2{ }^{\circ} \mathrm{C}\right.$, referring to the evaporation of the adsorbed water content), while this peak is not present in the DSC-curves of the $80 \% \mathrm{MMT} / \mathrm{S}$ composite and S, indicating the increased hydrophobicity. Besides, as $S$ content increases, the characteristic peaks of $S$ appear in the curves: the $\alpha \rightarrow \beta$ transition gives an endothermic peak at $\sim 110{ }^{\circ} \mathrm{C}$, while the endothermic melting is displayed at the $115-120^{\circ} \mathrm{C}$ range [24]. Upon cooling, the exothermic peaks of sulfur solidification and/or recrystallization are also presented [24].

As evidenced by SEM-images and energy-dispersive X-ray spectroscopy (EDX) measurements, the surface of the MMT/S composite with 80 $\mathrm{wt} \% \mathrm{~S}$ content is microstructured (Fig. 7.) and have a relatively 
A

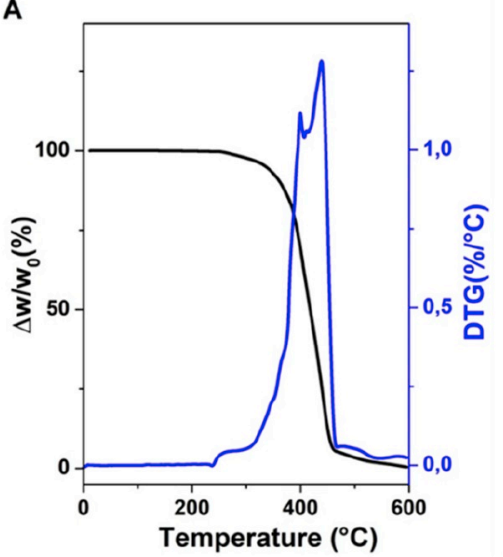

B

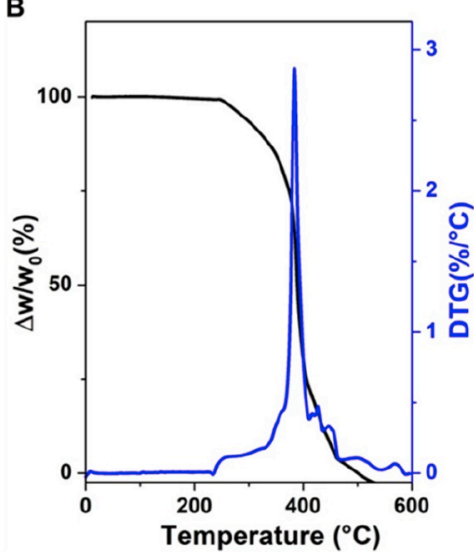

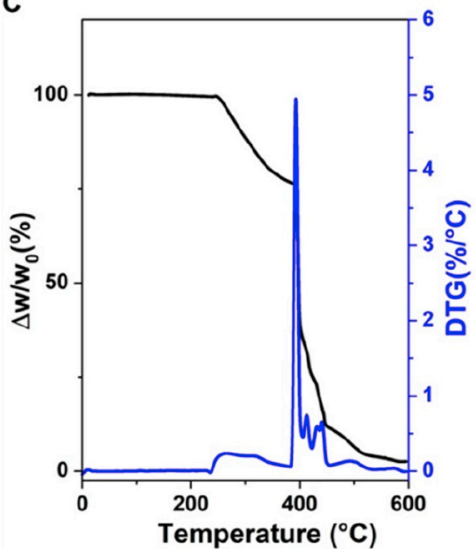

Fig. 11. TGA curves of (A) C-PE (B) C-PE-P (C) C-PE-C.

Table 2

Melting point and crystallization temperature of the PE composites.

\begin{tabular}{lll}
\hline Samples & Melting point $\left[{ }^{\circ} \mathrm{C}\right]$ & Crystallization temp. $\left[{ }^{\circ} \mathrm{C}\right]$ \\
\hline C-PE & 133.7 & 112.9 \\
C-PE-P & 129.3 & 115.9 \\
C-PE-C & 129.0 & 116.2 \\
\hline
\end{tabular}

homogenous and even distribution of both components.

The elemental composition was also determined based on EDXspectral data: as Fig. $7 \mathrm{~b}$ shows, the measured $75.45 \pm 5.95 \%$ sulfurcontent relatively well matches the nominal $80 \mathrm{wt} \%$. These observations indicate even and nearly lossless electrostatic deposition of S NPs with 1-40 nm primary particle size on the surface of barrier MMT lamellae platforms with lateral size of about few hundred nanometers.

To conclude the results, we managed to hydrophobize the MMT lamellae by fixing water insoluble and inorganic S NPs on their surface utilizing electrostatic interactions. The hydrophobicity of the composites sharply increased at $80 \% \mathrm{~S}$ content, while the dispersibility in water and zeta potential absolute values dropped at this composition.

Considering the above presented results at $80 \mathrm{wt} \% \mathrm{~S}$ content the MMT/S composite has relatively high surface charge (ca. $-11.6 \pm 1.8$ $\mathrm{mV}$ zeta potential) which is due to the accessible hydrophilic MMT surfaces and it also shows moderate hydrophobicity (WCA $=90.3 \pm$ $7.2^{\circ}$ ) because of the surface immobilized S NPs.

With the resulting amphyphylic character, the prepared composite particles could potentially be applied as Pickering-type emulsifiers or surfactants, as they may provide a more effective stabilization alternative than molecular surfactants due to their resistance to coalescence and Ostwald-ripening [25]. According to our idea this surfactant-like amphiphilic character of the inorganic composite could be useable for further plastic applications, because it could enhance the interfacial interactions between the different polymer filler materials and the continuous polymer matrix.

To study this assumption, the inorganic composite with $80 \mathrm{wt} \% \mathrm{~S}$ content was added to a commercially available PE with $0.5 \mathrm{wt} \% \mathrm{CB}$ content and the dispersion degree of the incorporated $\mathrm{CB}$ was

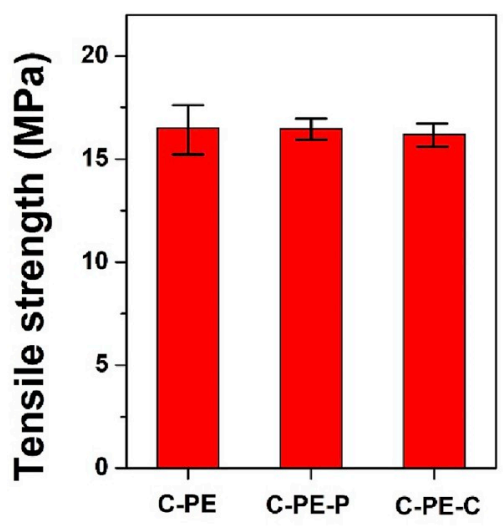

Fig. 13. Tensile strength of the samples.
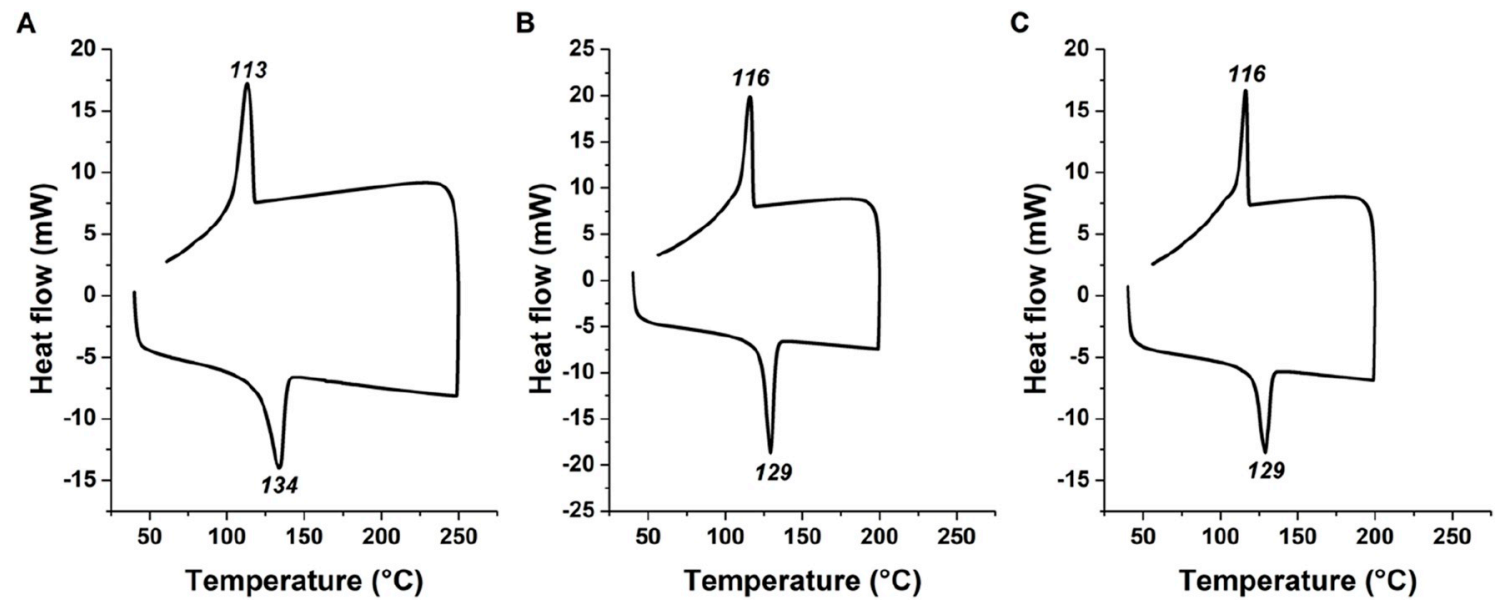

Fig. 12. DSC curves of (A) C-PE (B) C-PE-P (C) C-PE-C. 
characterized. As it is reported, many polymers are unsuitable for outdoor use on their own because of rapid degradation through the action of visible and especially UV light [26]. However, CB absorbs both visible and UV radiation converting it to heat and thus makes an excellent stabilizer. As the UV-VIS diffuse reflectance spectra of Fig. 8 show, the $0.5 \mathrm{wt} \% \mathrm{CB}-$ content in our samples has a considerable contribution to the UV-absorbance, as well. In many cases stabilities spanning decades can be obtained, making the polymers suitable for outdoor applications. UV stabilization by CB in polymers depends on its ability to absorb and to backscatter light. While the absorption increases with smaller particles and aggregates, the backscattering decreases and thus the UV stabilization requires small and evenly dispersed $C B$ particles in the continuous polymer matrix. Therefore, the effect of MMT/S surfactant-like additive on the dispersion degree of CB in HDPE polymer matrix was investigated.

The 3-dimensional structure of the polymers and the size distribution of the embedded carbon particles were studied by computed tomography (micro-CT) measurement. The representative CT-images in Fig. 9 show the incorporated CB particles in HDPE matrix. The corresponding particle size distributions of the examined specimens are presented in Fig. 10., while the average particle size values obtained are indicated in Table 1. As it appeared in the pictures and from the data, the average size of the particles are really similar in a case of the C-PE and C-PE-P samples, but in the case of the C-PE-C composite with the loading of the as-prepared MMT/S (with $80 \mathrm{wt} \%$ of S NP content) this value is became significantly smaller. In parallel, the average particle size was significantly decreased indicating higher distribution (small and evenly incorporated particles) of CB in HDPE. The difference between the C-PE$\mathrm{P}$ (simply physical mixture of S and MMT) and C-PE-C (electrostatically adhered S NPs on MMT) containing samples indicates that only this latter one behaves like a surfactant, the physical mixture is not suitable for increasing the dispersion degree of $\mathrm{CB}$. There are documented cases in the literature, which are in good accordance with our results: in an example, the addition of max $6.8 \%$ hydrophobized (di-methyl, dihydrogenated tallow-) MMT as a dispersing additive could increase the dispersion degree of multicomponent (matrix: polypropylene; disperse phase: ethylene-octene based elastomer) thermoplastics blends. The authors reported a decrease in the size of the dispersed elastomer particles with increasing MMT-content and fixed elastomer concentration: besides the amphyphylic properties, the addition of functionalized MMT resulted in viscosity build-up, which also contributed to the dispersibility-increase through the retardation of polymer-melt coalescence during mixing $[27,28]$. While the viscosity build-up is generally considered as a hindering factor during the blending process, in this case it can result in beneficially high dispersion degrees, as well.

In line with this, the particle density also changes, which nearly tripled in the C-PE-C sample compared to the C-PE or C-PE-P samples. (Table 1). These results suggest that the MMT/S nanocomposite (C-PEC) obviously increase the distribution of the carbon particles in the continuous polymer matrix and decrease their aggregation. This structure with evenly and continuously dispersed CB particles is advantageous in the viewpoint of UV stabilization of the polymer.

TGA was used to study the thermal stability of the HDPE composites (Fig. 11.). It was showed that pure HDPE is thermally stable up to 250 ${ }^{\circ} \mathrm{C}$. At $250{ }^{\circ} \mathrm{C}$ it starts to degrade and fully degraded at $470{ }^{\circ} \mathrm{C}$. Adding MMT/S additives to HDPE has no significant effect (Table 2), only a slight thermal stability was observed as the C-PE-P and C-PE-C samples started to decompose at $240{ }^{\circ} \mathrm{C}$.

DSC of HDPE shows an endothermic process (melting of the polymer) at $134{ }^{\circ} \mathrm{C}$ and an exothermic crystallization at $113{ }^{\circ} \mathrm{C}$ [29]. The DSC curves for C-PE-P and C-PE-C displayed slightly lower melting and higher crystallization temperature compared to the C-PE composite (Fig. 12.). This indicates that, the addition of MMT and S to the C-PE does not change appreciably the melting and crystallization temperature of the resulting polymer. Considering the related literature, the increase in crystallization temperature can be attributed to the nucleating effect of MMT and S [30].

The tensile properties of C-PE, C-PE-P, C-PE-C samples are shown in Fig. 13. The tests revealed that the additives did not significantly alter the tensile strength of HDPE in either case.

In addition to the introduced beneficial properties of the MMT/S polymer additive, it is important to mention, that the composite offers a way cheaper alternative to the commercially available organic surfactants. For instance, the current (2020 January) average bentonite clay and sulfur prices are at approximately 77 and $140 \mathrm{U} \mathrm{S}$. dollars per ton, respectively. The prices of the synthetic and bio-based organic surfactants depend on their type (anionic, non-ionic, cationic, and amphoteric) and applications (detergents, oil industry, personal care, textile, elastomers \& plastics, crop protection, food \& beverage, etc.), however, their price is at least one order of magnitude more expensive ( $>1100 \mathrm{U}$ S. dollars per ton). Furthermore, as elemental sulfur is an abundant byproduct of the petrochemical industry, and MMT is easily accessible, our composite can reduce the ecological footprint of thermoplastics production, as well.

\section{Conclusion}

Sulfur modified MMT with different compositions were blended with Carbon Black-containing HDPE composite by different methods. An electrostatically adhered S NPs on MMT composite with a sulfur loading of $80 \mathrm{wt} \%$ showed both hydrophobic and hydrophilic nature. This material as a surfactant-like amphiphilic additive was blended into Carbon Black-containing HDPE structure with a low (0.5 wt\%) loading concertation. In this case, the basic properties (heat stability, tensile strength) of the composite was not changed significantly, however, the carbon black additive distribution was increased drastically. The average particle size of the CB was 2 times smaller in the C-PE-C composite compared to the basic C-PE matrix. We believe that this method will be a helpful way to disperse additives in highly viscous hydrophobic or hydrophilic materials even at high temperature.

\section{Declaration of competing interest}

The authors declare that they have no known competing financial interests or personal relationships that could have appeared to influence the work reported in this paper.

\section{Acknowledgements}

This work was supported by the Hungarian National Research, Development and Innovation Office [GINOP-2.3.2-15-2016-00013 and GINOP-2.3.3-15-2016-00010], as well as the New National Excellence Program of the Ministry of Human Capacities [UNKP-19-3 and UNKP19-4]; the János Bolyai Research Scholarship of the Hungarian Academy of Sciences, and the Ministry of Human Capacities, Hungary [20391-3/ 2018/FEKUSTRAT and EFOP-3.6.1-16-2016-00014]. This paper was also supported by the Hungarian Research Development and Innovation Office through grants NKFIH OTKA PD 120877 of AS. AK, and KZ is grateful for the fund of NKFIH (OTKA) K112531 \& NN110676and K120115, respectively.

\section{Appendix A. Supplementary data}

Supplementary data to this article can be found online at https://doi. org/10.1016/j.polymertesting.2020.106419.

\section{References}

[1] M. Biswas, S.S. Ray, Recent progress in synthesis and evaluation of polymermontmorillonite nanocomposites, in: New Polymerization Techniques and Synthetic Methodologies, Springer, 2001, pp. 167-221. 
[2] A. Usuki, N. Hasegawa, M. Kato, S. Kobayashi, Polymer-clay nanocomposites, in: Inorganic Polymeric Nanocomposites and Membranes, Springer, 2005, pp. 135-195.

[3] Z.X. Huang, L.Y. Zhou, G.Z. Zhang, J.P. Qu, H.Z. He, Study on the properties of polyethylene/montmorillonite nanocomposites prepared by a novel vane mixer, J. Appl. Polym. Sci. 132 (2015).

[4] M. Kotal, A.K. Bhowmick, Polymer nanocomposites from modified clays: recent advances and challenges, Prog. Polym. Sci. 51 (2015) 127-187.

[5] T.T. Zhu, C.H. Zhou, F.B. Kabwe, Q.Q. Wu, C.S. Li, J.R. Zhang, Exfoliation of montmorillonite and related properties of clay/polymer nanocomposites, Appl. Clay Sci. 169 (2019) 48-66.

[6] A. Peacock, Handbook of Polyethylene: Structures: Properties, and Applications, CRC press, 2000.

[7] K. Ahmed, Synergistic effect of industrial waste in high density polyethylene, J. Mater. Environ. Sci. 5 (2014) 849-858.

[8] E. Tarani, A. Wurm, C. Schick, D. Bikiaris, K. Chrissafis, G. Vourlias, Effect of graphene nanoplatelets diameter on non-isothermal crystallization kinetics and melting behavior of high density polyethylene nanocomposites, Thermochim. Acta 643 (2016) 94-103.

[9] D.M. Nguyen, T.M.L. Nguyen, J. Colin, P. Perré, T.D. Nguyen, H.H. Thuc, C.N. H. Thuc, Monoglyceride as an effective and friendly modification agent for nanolayered structure of montmorillonite, Appl. Clay Sci. 179 (2019), 105100.

[10] G.W. Beall, M. Goss, Self-assembly of organic molecules on montmorillonite, Appl. Clay Sci. 27 (2004) 179-186.

[11] J.-J. Lin, Y.-N. Chan, Y.-F. Lan, Hydrophobic modification of layered clays and compatibility for epoxy nanocomposites, Materials 3 (2010) 2588-2605.

[12] P.P. von Weimarn, Kolloidchem. Beihefte 22 (1926) 38.

[13] M. Raffo, Kolloid Z. 2 (1908) 358.

[14] A. Gutbier, Thermische kolloidsynthesen, Z. Anorg. Allg. Chem. 152 (1926) 163.

[15] R. Steudel, Elemental sulfur, Top. Curr. Chem. 230 (2003) 153-166.

[16] A. Helmy, E. Ferreiro, S. De Bussetti, Cation exchange capacity and condition of zero charge of hydroxy-Al montmorillonite, Clay Clay Miner. 42 (1994) 444-450.

[17] P.-I. Au, Y.-K. Leong, Surface chemistry and rheology of slurries of kaolinite and montmorillonite from different sources, KONA Powder Particle J. (2016), 2016007.

[18] V. Krupskaya, S. Zakusin, E. Tyupina, O. Dorzhieva, A. Zhukhlistov, P. Belousov, M. Timofeeva, Experimental study of montmorillonite structure and transformation of its properties under treatment with inorganic acid solutions, Minerals 7 (2017) 49.

[19] A. Ahmed, Y. Chaker, E.H. Belarbi, O. Abbas, J. Chotard, H. Abassi, A.N. Van Nhien, M. El Hadri, S. Bresson, Xrd and Atr/Ftir investigations of various montmorillonite clays modified by monocationic and dicationic imidazolium ionic liquids, J. Mol. Struct. 1173 (2018) 653-664.

[20] A. Caron, J. Donohue, The X-ray powder pattern of rhombohedral sulfur, J. Phys. Chem. 64 (1960) 1767-1768.

[21] M. Biswas, S.S. Ray, Recent progress in synthesis and evaluation of polymermontmorillonite nanocomposites, in: New Polymerization Techniques and Synthetic Methodologies, Springer, 2001, pp. 167-221.

[22] Y. Zheng, A. Zaoui, I. Shahrour, Evolution of the interlayer space of hydrated montmorillonite as a function of temperature, Am. Mineral. 95 (10) (2010) 1493-1499.

[23] S.L. Teich-McGoldrick, J.A. Greathouse, C.F. Jové-Colón, R.T. Cygan, Swelling properties of montmorillonite and beidellite clay minerals from molecular simulation: comparison of temperature, interlayer cation, and charge location effects, J. Phys. Chem. C 119 (36) (2015) 20880-20891.

[24] B. Meyer, Elemental sulfur. http://web.gps.caltech.edu/ vijay/Papers/Che mistry/Meyer-76.pdf.

[25] L. Zhang, Q. Lei, J. Luo, M. Zeng, L. Wang, D. Huang, X. Wang, S. Mannan, B. Peng, Z. Cheng, Natural halloysites-based Janus platelet surfactants for the formation of pickering emulsion and enhanced oil recovery, Sci. Rep. 9 (1) (2019) 163.

[26] M.E. Spahr, R. Rothon, Carbon Black as a polymer filler, in: Encyclopedia of Polymers and Composites, Springer, 2015.

[27] H. Lee, P.D. Fasulo, W.R. Rodgers, D.R. Paul, TPO based nanocomposites. Part 1. Morphology and mechanical properties, Polymer 46 (25) (2005) 11673-11689.

[28] M.M. Vuksanović, R. Jančić Heinemann, Micro and nanoscale morphology characterization of compatibilized polymer blends by microscopy, in: Compatibilization of Polymer Blends, Elsevier, 2020, pp. 299-330.

[29] Q. Zhang, M.U. Khan, X. Lin, H. Cai, H. Lei, Temperature varied biochar as a reinforcing filler for high-density polyethylene composites, Compos. B Eng. 175 (2019), 107151.

[30] G. Liang, J. Xu, W. Xu, PE/PE-g-MAH/Org-MMT nanocomposites. II. Nonisothermal crystallization kinetics, J. Appl. Polym. Sci. 91 (5) (2004) 3054-3059. 